\title{
A new MEMS three-axial frequency-modulated (FM) gyroscope: a mechanical perspective
}

\author{
Valentina Zega ${ }^{\mathrm{a}}$, Claudia Comi ${ }^{\mathrm{a}}$, Paolo Minotti ${ }^{\mathrm{b}}$, Giacomo Langfelder ${ }^{\mathrm{b}}$, Luca \\ Falorni $^{\mathrm{c}}$, Alberto Corigliano ${ }^{\mathrm{a}, 1}$ \\ ${ }^{a}$ Department of Civil and Environmental Engineering, Politecnico di Milano, Italy \\ ${ }^{b}$ Department of Electronics, Information and Bioengineering, Politecnico di Milano, Italy \\ ${ }^{c} S T M i c r o e l e c t r o n i c s, A M G R \& D$
}

\begin{abstract}
Micro-Electro-Mechanical Systems (MEMS) gyroscopes are inertial sensors for the measurement of angular rates. They have a variety of applications from consumer electronics to drones and the need of stability against environmental fluctuations, such as temperature, is a key factor in order to avoid expensive calibration procedures.

Frequency Modulation (FM) has been recently proposed as an innovative working principle for MEMS gyroscopes and as the desired solution in terms of stability against environmental fluctuations.

In this paper, the FM working principle is formalized for the three-axial case for the first time and the governing equations are derived both in the idealized case of a point-mass gyroscope and in the real case of a distributed-mass gyroscope. Moreover, the mechanical structure of the first three-axial MEMS FM gyroscope is proposed and studied. Preliminary experimental measurements prove the validity of both the model and the simulations results employed during the design process. The proposed structure overcomes lots of the constraints of the surface micromachining fabrication processes and represents an important step towards the development of a new class of MEMS gyroscopes.
\end{abstract}

Keywords: MEMS, gyroscope, frequency-modulated (FM), mechanical design

\footnotetext{
* Corresponding author

Email address: alberto.corigliano@polimi.it (Alberto Corigliano)
} 
2010 MSC: 00-01, 99-00

\section{Introduction}

MEMS gyroscopes are micro-devices able to measure the external angular rate through the exploitation of different physical phenomena among which the Coriolis force is the most common one (see [1]). Depending on the component

5 of the external angular velocity that is measured, it is possible to distinguish between yaw, pitch and roll gyroscopes, see Figure 1a. A three-axial gyroscope is able to measure all the three components of the external angular velocity simultaneously.

A generic three-axes Coriolis based gyroscope can be modeled as a three degrees of freedom mass spring damper system that is observed in a rotating reference frame as shown in Figure $1 b$ for the case of a yaw gyroscope. The gyroscope proof mass is, in fact, contained inside a box (the MEMS box) and is required to be free to oscillate in all the three orthogonal directions. The Coriolis force, that arises because of the external angular velocity, couples the 15 degrees of freedom allowing the motion in a direction which is orthogonal to both the driving one and the one of the angular velocity. By measuring the effect of the Coriolis force on the system, the external angular velocity is detected. In Figure 1c, the working principle of a Coriolis based yaw gyroscope is shown: the mass is kept in oscillation in the $x$-direction and a Coriolis force acts in the $y$-direction as a consequence of the external angular rate $\Omega_{z}$.

Depending on the actuation/detection schemes employed, it is possible to distinguish between different kinds of MEMS gyroscopes. Amplitude Modulated (AM) MEMS gyroscopes (see e.g. [2] and [3]) rely on controlling the driving motion of the mass through a close-loop circuit and measuring the displacement induced by the Coriolis force. Almost all the MEMS gyroscopes available so far are based on this working principle and, despite a lot of work has been done in order to optimize the design of such devices in terms of sensitivity (see e.g. [4]), linearity (see e.g. [5] and [6]), bandwidth (see e.g. [7]), quadrature reduction 
a)
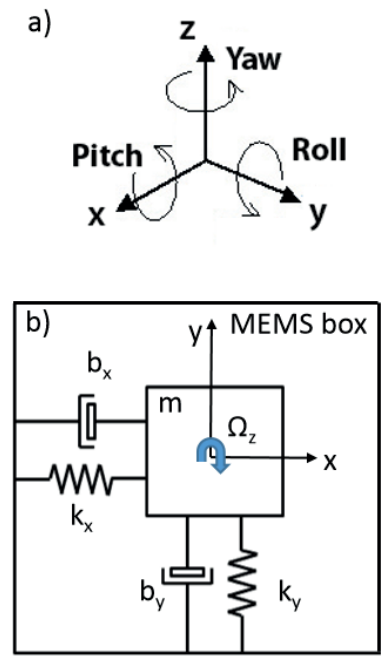

c)

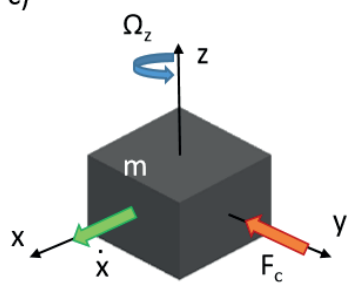

$\mathrm{F}_{\mathrm{c}}=-2 \mathrm{~m} \Omega_{\mathrm{z}} \wedge \dot{x}$

Figure 1: a) Notation, b) mass-spring-damper model: only the effects of a $z$-axis angular rate are reported for the sake of clarity, c) working principle of a yaw Coriolis based gyroscope. 
(see e.g. [8] and [9]) and stability (see e.g. [10] and [11]), there is still the need to calibrate each device with consequent high costs for the MEMS industry.

Recently, Frequency Modulated (FM) gyroscopes have been proposed (see e.g. [12]) as a possible solution toward the measurements stability against environmental fluctuations as temperature. Thanks to their stability, they promise to overcome the need of the calibration, thus reducing the costs and representing an innovative step toward a new class of MEMS gyroscopes. Instead of controlling the motion of one mode (the drive) and measuring the Coriolis-induced displacement amplitude variations along the three sense axes as done in AM solutions, FM gyroscopes rely on controlling the velocities of the three main orthogonal modes of the proof mass and in measuring the resonance frequency 40 variations induced by the external angular rate on the considered axes.

Yaw FM gyroscopes were firstly proposed and experimentally tested by the Berkeley Sensors and Actuators Center (see e.g. [13] and [14]), while pitch and roll FM gyroscopes were presented for the first time by the authors in [15] and [16].

Here, the FM working principle presented in [17] is extended to the threeaxial case and the mechanical design of the first three-axial FM gyroscope is proposed. Preliminary experimental tests on the fabricated prototype confirm the simulated properties of the device. The mechanical structure here proposed represents an innovative solution in the MEMS field in terms of out-of-plane driving and sensing (see [18]).

The paper is organized as follow: in Section 2, the theoretical model describing the dynamics of a three-axial MEMS gyroscope in the ideal point mass case and in the case of distributed-mass with the presence of fabrication imperfections and non-idealities is shown; in Section 3 the governing dynamic equations are solved through the phasor analysis. Section 4 describes the FM working principle and the main properties of a FM gyroscope. In Section 5 the mechanical design of the first three-axial FM gyroscope is proposed while in Section 6 , the first experimental characterization confirms the predictions presented in Section 5. Finally, closing remarks are reported in Section 7. 
a)
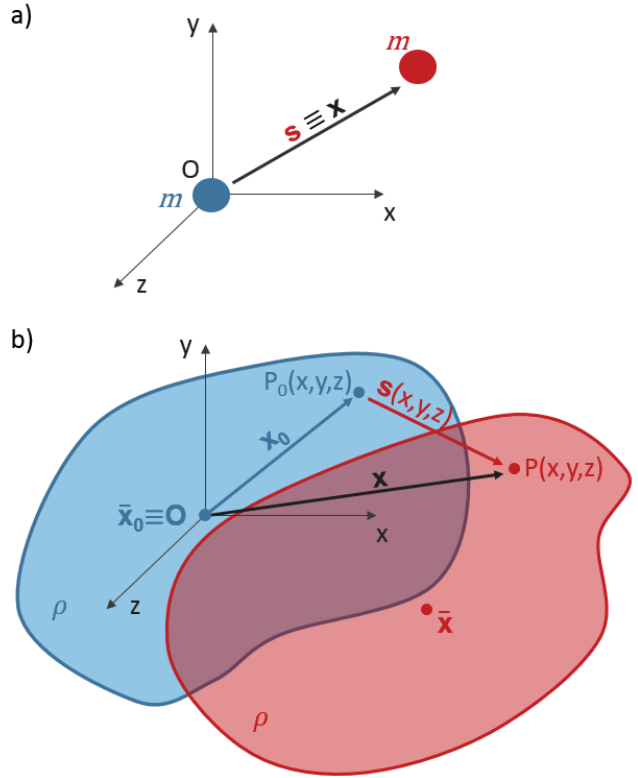

Figure 2: Reference system and notation for a) a point mass representing an idealized gyroscope and b) a 3D distributed-mass representing a real gyroscope.

\section{Dynamics of a MEMS Coriolis based gyroscope: theoretical model}

The equations of motion for the mass of a MEMS gyroscope can be derived through the use of relative dynamics, [1]. Note that, while the ideal gyroscope is a point mass structure suspended through three springs and three dampers, real gyroscopes are constituted by a non-point mass suspended through a complex system of springs and dampers, as it will be made clear in Section 5, when discussing the proposed innovative design for a three-axial FM gyroscope.

\subsection{Equations of motion for an ideal gyroscope}

Let us consider a pointwise mass $m$ initially located in the origin $O$ of a non-inertial frame. The mass is constrained by springs (see Figure 1b) and its displacement vector $\mathbf{s}$ coincides with its current position $\mathbf{x}$ (see Figure 2a). The local non-inertial frame translates with velocity $\mathbf{v}_{0}$ and rotates with angular velocity $\boldsymbol{\Omega}$ with respect to an inertial frame. 
The kinetic energy $\mathcal{T}$ and the potential energy $\mathcal{V}$ of the system are respectively:

$$
\begin{aligned}
& \mathcal{T}=\frac{1}{2} m \mathbf{v} \cdot \mathbf{v} \quad \text { with } \quad \mathbf{v}=\mathbf{v}_{0}+\dot{\mathbf{x}}+\boldsymbol{\Omega} \wedge \mathbf{x}, \\
& \mathcal{V}=\frac{1}{2} \mathbf{x} \cdot \mathbf{k} \mathbf{x}+\mathcal{V}_{\text {ext }},
\end{aligned}
$$

75 potential of external conservative forces $\mathbf{F}$ and a superposed dot denotes time derivative.

Denoting by $\mathcal{L}=\mathcal{T}-\mathcal{V}$ the Lagrangian functional, the equations of motion read:

$$
\frac{\mathrm{d}}{\mathrm{d} t} \frac{\partial \mathcal{L}}{\partial \dot{\mathbf{x}}}-\frac{\partial \mathcal{L}}{\partial \mathbf{x}}=\mathbf{Q},
$$

where $\mathbf{Q}$ is the vector of the generalized forces not arising from a potential (i.e. the damping forces - $\mathbf{b} \dot{\mathbf{x}}$ with $\mathbf{b}$ diagonal matrix of coefficients $b_{x}, b_{y}$ and $b_{z}$ ).

By substituting equations (1) in (2), the three-dimensional motion of a point mass gyroscope can be described through:

$$
\begin{array}{r}
m \ddot{\mathbf{x}}+m \dot{\boldsymbol{\Omega}} \wedge \mathbf{x}+2 m \boldsymbol{\Omega} \wedge \dot{\mathbf{x}}+m \boldsymbol{\Omega} \wedge \mathbf{v}_{0}+ \\
+m \mathbf{a}_{0}+m \boldsymbol{\Omega} \wedge \boldsymbol{\Omega} \wedge \mathbf{x}+\mathbf{k x}=\mathbf{F}-\mathbf{b} \dot{\mathbf{x}}
\end{array}
$$

where $\mathbf{a}_{0}=\dot{\mathbf{v}}_{0}$ is the external acceleration.

In the following, we will consider the external velocity $\mathbf{v}_{0}$ and acceleration $\mathbf{a}_{0}$ small enough to make the terms $m \boldsymbol{\Omega} \wedge \mathbf{v}_{0}$ and $m \mathbf{a}_{0}$ negligible in equation (3). For later use, one can also re-write the equations of motion (3) in the form:

$$
\begin{array}{r}
m \ddot{\mathbf{x}}+\mathbf{b} \dot{\mathbf{x}}+\mathbf{k x}+m\left(\boldsymbol{\alpha}^{x} \dot{\Omega}_{x}+\boldsymbol{\alpha}^{y} \dot{\Omega}_{y}+\boldsymbol{\alpha}^{z} \dot{\Omega}_{z}\right) \mathbf{x}+ \\
+2 m\left(\boldsymbol{\alpha}^{x} \Omega_{x}+\boldsymbol{\alpha}^{y} \Omega_{y}+\boldsymbol{\alpha}^{z} \Omega_{z}\right) \dot{\mathbf{x}}+m\left(\boldsymbol{\beta}^{x x} \Omega_{x}^{2}+\boldsymbol{\beta}^{y y} \Omega_{y}^{2}+\right. \\
+\boldsymbol{\beta}^{z z} \Omega_{z}^{2}+\left(\boldsymbol{\beta}^{x y}+\boldsymbol{\beta}^{y x}\right) \Omega_{x} \Omega_{y}+\left(\boldsymbol{\beta}^{x z}+\boldsymbol{\beta}^{z x}\right) \Omega_{x} \Omega_{z}+ \\
\left.+\left(\boldsymbol{\beta}^{y z}+\boldsymbol{\beta}^{z y}\right) \Omega_{y} \Omega_{z}\right) \mathbf{x}=\mathbf{F}
\end{array}
$$

where the matrices $\boldsymbol{\alpha}^{i}$ and $\boldsymbol{\beta}^{i j}$ have components:

$$
\alpha_{h k}^{i}=-\epsilon_{i h k},
$$


and

$$
\beta_{h k}^{i j}=\delta_{i h} \delta_{j k}-\delta_{i j} \delta_{h k},
$$

90 with $\delta_{h k}$ the Kronecker delta and $\epsilon_{i h k}$ the Levi-Civita symbol defined as:

$$
\epsilon_{i j k}=\left\{\begin{array}{cc}
1 & \text { if }(i, h, k)=(x, y, z),(y, z, x),(z, x, y) \\
-1 & \text { if }(i, h, k)=(z, y, x),(x, z, y),(y, x, z) \\
0 & \text { otherwise. }
\end{array}\right.
$$

Note that the matrices $\boldsymbol{\alpha}^{i}$ contain the so-called angular gains that quantify the coupling in terms of modal masses between the two modes coupled by the Coriolis force. For a pointwise mass structure like the one considered in this Section, they are equal to one in modulus, but in case of real gyroscopes with distributed mass, like the ones studied in the following, they will be smaller than one and will play a crucial role.

By assuming resonant frequencies $\omega_{o i}^{2}=k_{i} / m$ much larger than the applied rate signals $\Omega_{i}$ and supposing only slow changes of $\Omega_{i}$, it is possible to neglect all the terms coming from the drag acceleration in equation (4) and consider only the Coriolis force that couples the three components of motion of the gyroscope proof mass. Equation (4), then, reads:

$$
m \ddot{\mathbf{x}}+\mathbf{b} \dot{\mathbf{x}}+\mathbf{k x}+2 m\left(\boldsymbol{\alpha}^{x} \Omega_{x}+\boldsymbol{\alpha}^{y} \Omega_{y}+\boldsymbol{\alpha}^{z} \Omega_{z}\right) \dot{\mathbf{x}}=\mathbf{F} .
$$

\subsection{Equations of motion for a real gyroscope}

Real fabricated gyroscopes cannot be approximated as point mass structures because of their complex mechanical designs made by rigid masses and deformable structural elements, endowed with non-negligible mass, properly combined. The effect of all deformable elements (or springs) can be lumped in equivalent springs acting at the centroid $\overline{\mathbf{x}}$ of the device. Similarly, the nonconservative forces can be described by an equivalent damping matrix.

Moreover, real gyroscopes are affected by fabrication imperfections (i.e. small asymmetries in the deformable elements), as a consequence, it is no more possible to consider the three translational motions of the mass as decoupled. Extradiagonal terms in the equivalent stiffness and damping matrices are added to 
take into account such fabrication imperfections and consequent motion coupling.

The first three real modes of the structure, which are designed to be three ideal pure translational modes in the directions $x, y$ and $z$, are actually characterized by non-uniform displacement fields, s (see Figure $2 b$ ), that are here represented as:

$$
\mathbf{s}(x, y, z, t)=\left[\begin{array}{l}
\varphi^{x}(x, y, z) u_{x}(t) \\
\varphi^{y}(x, y, z) u_{y}(t) \\
\varphi^{z}(x, y, z) u_{z}(t)
\end{array}\right]=\varphi(\mathbf{x}) \mathbf{u}(t)
$$

where $\varphi(\mathrm{x})$ is the diagonal matrix containing the three nondimensional modal shape functions $\varphi^{x}, \varphi^{y}, \varphi^{z}$ describing the three main modes actuated by on board actuators in a real gyroscope (see Section 5$)$ and $\mathbf{u}^{T}(t)=\left[u_{x}(t), u_{y}(t), u_{z}(t)\right]$ is the time evolution of the system's response with $u_{x}, u_{y}$ and $u_{z}$ the amplitudes of the three modes. Note that, rotational degrees of freedom will not be included in the present formulation. This simplification is justified by the assumption that a proper mechanical design would allow to obtain rotational modes at very high frequencies with respect to those of the translational modes.

Denoting by $\rho$ the density of the distributed mass with volume $V$, the kinetic and potential energy of the real gyroscope read:

$$
\begin{aligned}
& \mathcal{T}=\frac{1}{2} \int_{V} \rho \mathbf{v} \cdot \mathbf{v d} V \quad \text { with } \quad \mathbf{v}=\mathbf{v}_{0}+\dot{\mathbf{s}}+\boldsymbol{\Omega} \wedge\left(\mathbf{s}+\mathbf{x}_{0}\right), \\
& \mathcal{V}=\frac{1}{2} \mathbf{s}(\overline{\mathbf{x}}) \cdot \mathbf{k}_{e q} \mathbf{s}(\overline{\mathbf{x}})+\mathcal{V}_{\text {ext }},
\end{aligned}
$$

where $\mathbf{k}_{e q}$ is the non-diagonal equivalent stiffness matrix. The vector of the 130 generalized forces $\mathbf{Q}$ is constituted by the damping forces $-\mathbf{b}_{e q} \dot{\mathbf{s}}(\overline{\mathbf{x}})$ with $\mathbf{b}_{e q}$ equivalent damping matrix that takes into account the fabrication imperfections through extra-diagonal terms.

Neglecting again contributions coming from the external velocity and acceleration, by substituting equations (10) into (2), the equations of motion of a 
real three-axial gyroscope are derived. They read:

$$
\begin{array}{r}
\mathbf{m} \ddot{\mathbf{u}}+\mathbf{b u}+\mathbf{k u}+\left(\dot{\Omega}_{x} \hat{\boldsymbol{\alpha}}^{x}+\dot{\Omega}_{y} \hat{\boldsymbol{\alpha}}^{y}+\dot{\Omega}_{z} \hat{\boldsymbol{\alpha}}^{z}\right) \mathbf{u}+2\left(\Omega_{x} \hat{\boldsymbol{\alpha}}^{x}+\right. \\
\left.+\Omega_{y} \hat{\boldsymbol{\alpha}}^{y}+\Omega_{z} \hat{\boldsymbol{\alpha}}^{z}\right) \dot{\mathbf{u}}+\left(\Omega_{x}^{2} \hat{\boldsymbol{\beta}}^{x x}+\Omega_{y}^{2} \hat{\boldsymbol{\beta}}^{y y}+\Omega_{z}^{2} \hat{\boldsymbol{\beta}}^{z z}+\Omega_{x} \Omega_{y}\left(\hat{\boldsymbol{\beta}}^{x y}+\right.\right. \\
\left.\left.+\hat{\boldsymbol{\beta}}^{y x}\right)+\Omega_{x} \Omega_{z}\left(\hat{\boldsymbol{\beta}}^{x z}+\hat{\boldsymbol{\beta}}^{z x}\right)+\Omega_{y} \Omega_{z}\left(\hat{\boldsymbol{\beta}}^{y z}+\hat{\boldsymbol{\beta}}^{z y}\right)\right) \mathbf{u}=\mathbf{F}
\end{array}
$$

where, $\mathbf{m}$ is the diagonal mass matrix whose non-zero elements are the modal masses of the three modes of interest, $\mathbf{k}$ is the stiffness matrix that contains extra-diagonal terms coming from the fabrication imperfections, $\hat{\boldsymbol{\alpha}}^{i}$ and $\hat{\boldsymbol{\beta}}^{i j}$, with $i, j=x, y, z$, are the coupling matrices that arise because of the presence of the external angular velocities.

The matrices $\mathbf{m}, \mathbf{b}$ and $\mathbf{k}$ have components $m_{h k}, b_{h k}$ and $k_{h k}$ respectively with $h$ and $k$ taking values $x, y$ and $z$. The terms of the matrices $\mathbf{m}, \mathbf{b}, \mathbf{k}, \hat{\boldsymbol{\alpha}}$ and $\hat{\boldsymbol{\beta}}$ are defined as:

$$
\begin{array}{lr}
m_{h k}=\int_{V} \rho \varphi^{h} \varphi^{k} \delta_{h k} \mathrm{~d} V & h, k=x, y, z \\
b_{h k}=\varphi^{h}(\overline{\mathbf{x}})\left(b_{e q}\right)_{h k} \varphi^{k}(\overline{\mathbf{x}}) & h, k=x, y, z \\
k_{h k}=\varphi^{h}(\overline{\mathbf{x}})\left(k_{e q}\right)_{h k} \varphi^{k}(\overline{\mathbf{x}}) & h, k=x, y, z \\
\hat{\alpha}_{h k}^{i}=-\int_{V} \rho \epsilon_{i h k} \varphi^{h} \varphi^{k} \mathrm{~d} V & i, h, k=x, y, z \\
\hat{\beta}_{h k}^{i j}=\int_{V} \rho\left(\varphi^{h} \varphi^{k} \delta_{i h} \delta_{j k}-\delta_{i j} \delta_{h k}\left(\varphi^{h}\right)^{2}\right) \mathrm{d} V & i, j, h, k=x, y, z
\end{array}
$$

where the summation over repeated indices is not considered.

The angular gain $\alpha_{h k}^{i}$ with $i, h, k=x, y, z$ of the distributed-mass gyroscope is defined as the ratio between $\hat{\alpha}_{h k}^{i}$ and the modal mass $m_{h h}$ as:

$$
\alpha_{h k}^{i}=\frac{\hat{\alpha}_{h k}^{i}}{m_{h h}} \quad(\text { distributed }- \text { mass }) \quad i, h, k=x, y, z .
$$

Note that in the limit of pointwise mass $\varphi^{h} \rightarrow 1, m_{h h} \rightarrow m$ and equation (13) coincides with equation (5). In general, since $\varphi^{h}$ vary in space, the entries of the angular gain matrices $\boldsymbol{\alpha}^{i}$ differ from unit.

Finally, note that the external velocities and accelerations can be neglected even when the external acceleration is not negligible; it is possible in fact to null 
their contributions through the design of a differential mechanical structure as shown in [19].

\section{Phasor analysis}

The equations of motion presented in the previous section are, here, solved through the phasor method (see [13]) both for the case of an ideal point-mass gyroscope and for a real gyroscope.

\subsection{Ideal Gyroscope}

Accounting for relations (5)-(6), the vectorial equation (4) can be written in the scalar form:

$$
\begin{array}{r}
m \ddot{x}+b_{x} \dot{x}-2 m \Omega_{z} \dot{y}+2 m \Omega_{y} \dot{z}+k_{x} x-m\left(\Omega_{z}^{2}+\Omega_{y}^{2}\right) x+ \\
+m\left(\Omega_{x} \Omega_{y}-\dot{\Omega}_{z}\right) y+m\left(\dot{\Omega}_{y}+\Omega_{x} \Omega_{z}\right) z=F_{x}, \\
m \ddot{y}+b_{y} \dot{y}-2 m \Omega_{x} \dot{z}+2 m \Omega_{z} \dot{x}+k_{y} y-m\left(\Omega_{z}^{2}+\Omega_{x}^{2}\right) y+ \\
+m\left(\Omega_{x} \Omega_{y}+\dot{\Omega}_{z}\right) x+m\left(\Omega_{z} \Omega_{y}-\dot{\Omega}_{x}\right) z=F_{y}, \\
m \ddot{z}+b_{z} \dot{z}-2 m \Omega_{y} \dot{x}+2 m \Omega_{x} \dot{y}+k_{z} z-m\left(\Omega_{x}^{2}+\Omega_{y}^{2}\right) z+ \\
+m\left(\Omega_{x} \Omega_{z}-\dot{\Omega}_{y}\right) x+m\left(\dot{\Omega}_{x}+\Omega_{y} \Omega_{z}\right) y=F_{z} .
\end{array}
$$

In order to make clearer the link between the results for the ideal gyroscope and those for a real one, we keep here the notation with $\alpha_{h k}^{i}$ and $\beta_{h k}^{i j}$, without substituting their values given in (5)-(6). Equations (14) will hence be written in the form:

$$
\begin{array}{r}
m \ddot{x}+b_{x} \dot{x}+2 m \alpha_{x y}^{z} \Omega_{z} \dot{y}+2 m \alpha_{x z}^{y} \Omega_{y} \dot{z}+k_{x} x+m\left(\beta_{x}^{z} \Omega_{z}^{2}+\beta_{x}^{y} \Omega_{y}^{2}\right) x+ \\
+m\left(\beta_{x y}^{x y} \Omega_{x} \Omega_{y}+\alpha_{x y}^{z} \dot{\Omega}_{z}\right) y+m\left(\beta_{x z}^{x z} \Omega_{x} \Omega_{z}+\alpha_{x z}^{y} \dot{\Omega}_{y}\right) z=F_{x}, \\
m \ddot{y}+b_{y} \dot{y}+2 m \alpha_{y z}^{x} \Omega_{x} \dot{z}+2 m \alpha_{y x}^{z} \Omega_{z} \dot{x}+k_{y} y+m\left(\beta_{y}^{z} \Omega_{z}^{2}+\beta_{y}^{x} \Omega_{x}^{2}\right) y+ \\
+m\left(\beta_{y x}^{y x} \Omega_{x} \Omega_{y}+\alpha_{y x}^{z} \dot{\Omega}_{z}\right) x+m\left(\beta_{y z}^{y z} \Omega_{z} \Omega_{y}+\alpha_{y z}^{x} \dot{\Omega}_{x}\right) z=F_{y}, \\
m \ddot{z}+b_{z} \dot{z}+2 m \alpha_{z x}^{y} \Omega_{y} \dot{x}+2 m \alpha_{z y}^{x} \Omega_{x} \dot{y}+k_{z} z+m\left(\beta_{z}^{x} \Omega_{x}^{2}+\beta_{z}^{y} \Omega_{y}^{2}\right) z+ \\
+m\left(\beta_{z x}^{z x} \Omega_{x} \Omega_{z}+\alpha_{z x}^{y} \dot{\Omega}_{y}\right) x+m\left(\beta_{z y}^{z y} \Omega_{y} \Omega_{z}+\alpha_{z y}^{x} \dot{\Omega}_{x}\right) y=F_{z},
\end{array}
$$



sake of clarity.

In order to compensate for losses and to sustain the oscillation of the gyroscope proof mass according to the three translational modes of interest, the excitation forces can be written in the form $\mathbf{F}^{T}=\left[\left(\mathrm{i} \bar{F}_{x}\right) e^{\mathrm{i} \phi_{x}},\left(\mathrm{i} \bar{F}_{y}\right) e^{\mathrm{i} \phi_{y}},\left(\mathrm{i} \bar{F}_{z s}\right) e^{\mathrm{i} \phi_{z}}\right]$ of the form:

$$
\boldsymbol{u}=\left[\begin{array}{l}
u_{x} \\
u_{y} \\
u_{z}
\end{array}\right]=\left[\begin{array}{c}
A_{x} e^{\mathrm{i} \phi_{x}} \\
A_{y} e^{\mathrm{i} \phi_{y}} \\
A_{z} e^{\mathrm{i} \phi_{z}}
\end{array}\right],
$$

where $A_{x}, A_{y}, A_{z}, \phi_{x}=\int_{0}^{t} \omega_{x}(\tau) \mathrm{d} \tau, \phi_{y}=\int_{0}^{t} \omega_{y}(\tau) \mathrm{d} \tau$ and $\phi_{z}=\int_{0}^{t} \omega_{z}(\tau) \mathrm{d} \tau$ represent the system unknowns. $A_{x}, A_{y}, A_{z}$ are the real, time dependent amplitudes of the displacements of the proof mass along the three directions $x, y$ and $z$, while $\omega_{x}, \omega_{y}$ and $\omega_{z}$ are the actual resonant frequencies of the three translational modes along the $x-, y$ - and $z$-directions. If no external angular rate is applied, $\omega_{i}=\omega_{o i}$ with $\omega_{o i}$ natural frequency of the $i$-mode and $\phi_{i}=\omega_{o i} t+\psi_{i}$. In the following we introduce the notation $\Delta \phi_{i j}=\phi_{i}-\phi_{j}$ with $i=x, y, z$.

Note that $A_{x}, A_{y}, A_{z}, \omega_{x}, \omega_{y}$ and $\omega_{z}$ are slowly varying relatively to the mechanical resonant frequencies of the system, as a consequence, in the acceleration expression, the terms containing $\ddot{A}_{x}, \ddot{A}_{y}, \ddot{A}_{z}, \dot{\omega}_{x}, \dot{\omega}_{y}$ and $\dot{\omega}_{z}$ can be neglected, as they are negligible if compared to $\omega_{x} \dot{A}_{x}, \omega_{y} \dot{A}_{y}, \omega_{z} \dot{A}_{z}, \omega_{x}^{2}, \omega_{y}^{2}$ and $\omega_{z}^{2}$, respectively. Moreover, it is reasonable to assume that the angular rate and the displacement amplitudes are slowly varying with respect to the resonance frequency.

Under these hypotheses, by substituting equations (16) into (15) and dividing the three equations for $e^{\mathrm{i} \phi_{x}}, e^{\mathrm{i} \phi_{y}}$ and $e^{\mathrm{i} \phi_{z}}$ respectively, three complex 
equations are obtained. By nulling both the real and the imaginary parts, it is possible to obtain an expression for $\dot{A}_{x}, \dot{A}_{y}, \dot{A}_{z}, \omega_{x}^{2}, \omega_{y}^{2}$ and $\omega_{z}^{2}$.

The expressions for $\dot{A}_{x}, \dot{A}_{y}$ and $\dot{A}_{z}$ are reported in Appendix A since they will not be used in the following, while the expressions for $\omega_{x}^{2}, \omega_{y}^{2}$ and $\omega_{z}^{2}$ under the assumption $\dot{A}_{i} \ll A_{i} \omega_{i}$ for $i=x, y, z$ read:

$$
\begin{array}{r}
\omega_{x}^{2}=\omega_{o x}^{2}+\beta_{x}^{z} \Omega_{z}^{2}+\beta_{x}^{y} \Omega_{y}^{2}+ \\
+\beta_{x y}^{x y} \Omega_{x} \Omega_{y} \frac{A_{y}}{A_{x}} \cos \left(\Delta \phi_{x y}\right)+\beta_{x z}^{x z} \Omega_{x} \Omega_{z} \frac{A_{z}}{A_{x}} \cos \left(\Delta \phi_{x z}\right)+ \\
+2 \alpha_{x y}^{z} \Omega_{z} \omega_{y} \frac{A_{y}}{A_{x}} \sin \left(\Delta \phi_{x y}\right)+2 \alpha_{x z}^{y} \Omega_{y} \omega_{z} \frac{A_{z}}{A_{x}} \sin \left(\Delta \phi_{x z}\right), \\
\omega_{y}^{2}=\omega_{o y}^{2}+\beta_{y}^{x} \Omega_{x}^{2}+\beta_{y}^{z} \Omega_{z}^{2}+ \\
+\beta_{y x}^{y x} \Omega_{x} \Omega_{y} \frac{A_{x}}{A_{y}} \cos \left(\Delta \phi_{x y}\right)+\beta_{y z}^{y z} \Omega_{y} \Omega_{z} \frac{A_{z}}{A_{y}} \cos \left(\Delta \phi_{y z}\right)+ \\
-2 \alpha_{y x}^{z} \Omega_{z} \omega_{x} \frac{A_{x}}{A_{y}} \sin \left(\Delta \phi_{x y}\right)+2 \alpha_{y z}^{x} \Omega_{x} \omega_{z} \frac{A_{z}}{A_{y}} \sin \left(\Delta \phi_{y z}\right), \\
\omega_{z}^{2}=\omega_{o z}^{2}+\beta_{z}^{x} \Omega_{x}^{2}+\beta_{z}^{y} \Omega_{y}^{2}+ \\
+\beta_{z y}^{z y} \Omega_{y} \Omega_{z} \frac{A_{y}}{A_{z}} \cos \left(\Delta \phi_{y z}\right)+\beta_{z x}^{z x} \Omega_{x} \Omega_{z} \frac{A_{x}}{A_{z}} \cos \left(\Delta \phi_{x z}\right)+ \\
-2 \alpha_{z x}^{y} \Omega_{y} \omega_{x} \frac{A_{x}}{A_{z}} \sin \left(\Delta \phi_{x z}\right)-2 \alpha_{z y}^{x} \Omega_{x} \omega_{y} \frac{A_{y}}{A_{z}} \sin \left(\Delta \phi_{y z}\right) .
\end{array}
$$

By defining the velocity amplitudes as $v_{i}=A_{i} \omega_{i}$ for $i=x, y, z$ and solving the second order equation (17) with respect to $\omega_{x}$, the following expression is found:

$$
\begin{array}{r}
\omega_{x}=\frac{\beta_{x y}^{x y}}{2 \omega_{o y}} \Omega_{x} \Omega_{y} \frac{v_{y}}{v_{x}} \cos \left(\Delta \phi_{x y}\right)+ \\
+\frac{\beta_{x z}^{x z}}{2 \omega_{o z}} \Omega_{x} \Omega_{z} \frac{v_{z}}{v_{x}} \cos \left(\Delta \phi_{x z}\right)+\alpha_{x y}^{z} \Omega_{z} \frac{v_{y}}{v_{x}} \sin \left(\Delta \phi_{x y}\right)+ \\
+\alpha_{x z}^{y} \Omega_{y} \frac{v_{z}}{v_{x}} \sin \left(\Delta \phi_{x z}\right)+\sqrt{\omega_{o x}^{2}+D_{x}}
\end{array}
$$

where

$$
\begin{array}{r}
D_{x}=\beta_{x}^{z} \Omega_{z}^{2}+\beta_{x}^{y} \Omega_{y}^{2}+\left(\frac{\beta_{x y}^{x y}}{2 \omega_{o y}} \Omega_{x} \Omega_{y} \frac{v_{y}}{v_{x}} \cos \left(\Delta \phi_{x y}\right)+\right. \\
+\frac{\beta_{x z}^{x z}}{2 \omega_{o z}} \Omega_{x} \Omega_{z} \frac{v_{z}}{v_{x}} \cos \left(\Delta \phi_{x z}\right)+\alpha_{x y}^{z} \Omega_{z} \frac{v_{y}}{v_{x}} \sin \left(\Delta \phi_{x y}\right)+ \\
\left.+\alpha_{x z}^{y} \Omega_{y} \frac{v_{z}}{v_{x}} \sin \left(\Delta \phi_{x z}\right)\right)^{2} .
\end{array}
$$


By proceeding in the same way for the other two axes and by noting that ${ }_{205} \omega_{o i}^{2} \gg D_{i}$ for $i=x, y, z$, the instantaneous frequencies of oscillation along the $x$-, $y$ - and $z$-axis are derived:

$$
\begin{array}{r}
\omega_{x}=\omega_{o x}+\frac{\beta_{x y}^{x y}}{2 \omega_{o y}} \Omega_{x} \Omega_{y} \frac{v_{y}}{v_{x}} \cos \left(\Delta \phi_{x y}\right)+ \\
+\frac{\beta_{x z}^{x z}}{2 \omega_{o z}} \Omega_{x} \Omega_{z} \frac{v_{z}}{v_{x}} \cos \left(\Delta \phi_{x z}\right)+ \\
+\alpha_{x y}^{z} \Omega_{z} \frac{v_{y}}{v_{x}} \sin \left(\Delta \phi_{x y}\right)+\alpha_{x z}^{y} \Omega_{y} \frac{v_{z}}{v_{x}} \sin \left(\Delta \phi_{x z}\right), \\
\omega_{y}=\omega_{o y}+\frac{\beta_{y x}^{y x}}{2 \omega_{o x}} \Omega_{x} \Omega_{y} \frac{v_{x}}{v_{y}} \cos \left(\Delta \phi_{x y}\right)+ \\
+\frac{\beta_{y z}^{y z}}{2 \omega_{o z}} \Omega_{y} \Omega_{z} \frac{v_{z}}{v_{y}} \cos \left(\Delta \phi_{y z}\right)+ \\
+\alpha_{y z}^{x} \Omega_{x} \frac{v_{z}}{v_{y}} \sin \left(\Delta \phi_{y z}\right)-\alpha_{y x}^{z} \Omega_{z} \frac{v_{x}}{v_{y}} \sin \left(\Delta \phi_{x y}\right), \\
\omega_{z}=\omega_{o z}+\frac{\beta_{z x}^{z x}}{2 \omega_{o x}} \Omega_{x} \Omega_{z} \frac{v_{x}}{v_{z}} \cos \left(\Delta \phi_{x z}\right)+ \\
+\frac{\beta_{z y}^{z y}}{2 \omega_{o y}} \Omega_{y} \Omega_{z} \frac{v_{y}}{v_{z}} \cos \left(\Delta \phi_{y z}\right)+ \\
-\alpha_{z x}^{y} \Omega_{y} \frac{v_{x}}{v_{z}} \sin \left(\Delta \phi_{x z}\right)-\alpha_{z y}^{x} \Omega_{x} \frac{v_{y}}{v_{z}} \sin \left(\Delta \phi_{y z}\right) .
\end{array}
$$

\subsection{Real Gyroscopes}

210

By applying the same procedure to the equation of motion (11) of a real gyroscope, the following expressions are obtained:

$$
\begin{aligned}
\omega_{x} & =\omega_{o x}+\left(\frac{k_{x y}}{2 \omega_{o y} m_{x}}+\frac{\hat{\beta}_{x y}^{x y}}{2 \omega_{o y} m_{x}} \Omega_{x} \Omega_{y}\right) \frac{v_{y}}{v_{x}} \cos \left(\Delta \phi_{x y}\right)+ \\
& +\left(\frac{k_{x z}}{2 \omega_{o z} m_{x}}+\frac{\hat{\beta}_{x z}^{x z}}{2 \omega_{o z} m_{x}} \Omega_{x} \Omega_{z}\right) \frac{v_{z}}{v_{x}} \cos \left(\Delta \phi_{x z}\right)+ \\
& +\left(\frac{b_{x y}}{2 m_{x}}+\alpha_{x y}^{z} \Omega_{z}\right) \frac{v_{y}}{v_{x}} \sin \left(\Delta \phi_{x y}\right)+ \\
& +\left(\frac{b_{x z}}{2 m_{x}}+\alpha_{x z}^{y} \Omega_{y}\right) \frac{v_{z}}{v_{x}} \sin \left(\Delta \phi_{x z}\right)
\end{aligned}
$$




$$
\begin{aligned}
\omega_{y}= & \omega_{o y}+\left(\frac{k_{y x}}{2 \omega_{o x} m_{y}}+\frac{\hat{\beta}_{y x}^{y x}}{2 m_{y} \omega_{o x}} \Omega_{x} \Omega_{y}\right) \frac{v_{x}}{v_{y}} \cos \left(\Delta \phi_{x y}\right)+ \\
& +\left(\frac{k_{y z}}{2 \omega_{o z} m_{y}}+\frac{\hat{\beta}_{y z}^{y z}}{2 m_{y} \omega_{o z}} \Omega_{y} \Omega_{z}\right) \frac{v_{z}}{v_{y}} \cos \left(\Delta \phi_{y z}\right)+ \\
& +\left(\frac{b_{y z}}{2 m_{y}}+\alpha_{y z}^{x} \Omega_{x}\right) \frac{v_{z}}{v_{y}} \sin \left(\Delta \phi_{y z}\right)+ \\
& -\left(\frac{b_{x y}}{2 m_{y}}+\alpha_{y x}^{z} \Omega_{z}\right) \frac{v_{x}}{v_{y}} \sin \left(\Delta \phi_{x y}\right), \\
\omega_{z}= & \omega_{o z}+\left(\frac{k_{z x}}{2 \omega_{o x} m_{z}}+\frac{\hat{\beta}_{z x}^{z x}}{2 m_{z} \omega_{o x}} \Omega_{x} \Omega_{z}\right) \frac{v_{x}}{v_{z}} \cos \left(\Delta \phi_{x z}\right)+ \\
& +\left(\frac{k_{y z}}{2 \omega_{o y} m_{z}}+\frac{\hat{\beta}_{z y}^{z y}}{2 m_{z} \omega_{o y}} \Omega_{y} \Omega_{z}\right) \frac{v_{y}}{v_{z}} \cos \left(\Delta \phi_{y z}\right)+ \\
& -\left(\alpha_{z x}^{y} \Omega_{y}+\frac{b_{x z}}{2 m_{z}}\right) \frac{v_{x}}{v_{z}} \sin \left(\Delta \phi_{x z}\right)+ \\
& -\left(\frac{b_{y z}}{2 m_{z}}+\alpha_{z y}^{x} \Omega_{x}\right) \frac{v_{y}}{v_{z}} \sin \left(\Delta \phi_{y z}\right),
\end{aligned}
$$

while the expressions describing the variation of the motion amplitudes are reported in Appendix A for the sake of completeness.

Equations (25)-(27) and equations (A.4)-(A.6) describe the dynamics of a generic Coriolis based three-axial MEMS gyroscope and, in particular, depending on the constraints and on the chosen free variables, they can refer to both the AM and the FM working principle (see [13] for more details).

\section{FM working principle}

The FM working principle is based on the control of the velocities of the proof mass along three orthogonal directions through an electronic circuit and on the measurement of the frequency variations of the three modes of interest induced by the Coriolis forces.

In a three-axial FM gyroscope, there are therefore three driven directions and the 'velocity amplitude relationship' reads:

$$
v_{x}=v_{y}=v_{z} .
$$


If the natural frequencies of the three translational modes of the FM gyroscope are different one from the others by design (i.e. there is a mismatch between the modes in the MEMS language), when no external angular rate is applied to the device, the mass oscillates according to:

$$
\begin{aligned}
& u_{x}=A_{x} \cos \left(\omega_{o x} t+\psi_{x}\right), \\
& u_{y}=A_{y} \cos \left(\omega_{o y} t+\psi_{y}\right), \\
& u_{z}=A_{z} \cos \left(\omega_{o z} t+\psi_{z}\right) .
\end{aligned}
$$

The trajectory described through equations (29) is known as 3D Lissajous trajectory (see [20]) and for this reason, these devices are referred as LFM threeaxial gyroscopes.

In order to make the equations describing the frequency modulation working principle easier to read and understand, the expressions (22)-(24) describing the dynamic behavior of an ideal gyroscope are considered. The ratios between the velocity amplitudes are equal to one under the hypothesis of FM working principle (see the relation (28)) and the terms proportional to the product of two components of the external angular rate are usually negligible with respect to the other terms:

$$
\begin{aligned}
& \omega_{x}=\omega_{o x}+\alpha_{x y}^{z} \Omega_{z} \sin \left(\Delta \phi_{x y}\right)+\alpha_{x z}^{y} \Omega_{y} \sin \left(\Delta \phi_{x z}\right), \\
& \omega_{y}=\omega_{o y}+\alpha_{y z}^{x} \Omega_{x} \sin \left(\Delta \phi_{y z}\right)-\alpha_{y x}^{z} \Omega_{z} \sin \left(\Delta \phi_{x y}\right), \\
& \omega_{z}=\omega_{o z}-\alpha_{z x}^{y} \Omega_{y} \sin \left(\Delta \phi_{x z}\right)-\alpha_{z y}^{x} \Omega_{x} \sin \left(\Delta \phi_{y z}\right) .
\end{aligned}
$$

Equations (30) state that the components of the external angular rate $\Omega_{x}$, $\Omega_{y}$ and $\Omega_{z}$ modulate the frequencies of the three modes of the gyroscope through the factor $\alpha_{h k}^{i}$ with $i, h, k=x, y, z$.

The scale factors or sensitivities of a FM gyroscope are defined as the variation of the resonance frequency induced by the external angular rate and, for 
the ideal case, can be computed as:

$$
\begin{aligned}
& \frac{\partial \omega_{\Sigma}}{\partial \Omega_{x}}=+\alpha_{y z}^{x} \sin \left(\Delta \phi_{y z}\right)-\alpha_{z y}^{x} \sin \left(\Delta \phi_{y z}\right), \\
& \frac{\partial \omega_{\Sigma}}{\partial \Omega_{y}}=-\alpha_{z x}^{y} \sin \left(\Delta \phi_{x z}\right)+\alpha_{x z}^{y} \sin \left(\Delta \phi_{x z}\right), \\
& \frac{\partial \omega_{\Sigma}}{\partial \Omega_{z}}=+\alpha_{x y}^{z} \sin \left(\Delta \phi_{x y}\right)-\alpha_{y x}^{z} \sin \left(\Delta \phi_{x y}\right),
\end{aligned}
$$

where $\omega_{\Sigma}=\omega_{x}+\omega_{y}+\omega_{z}$.

A properly designed electronic circuit (see [21] for more details) manipulates the output signals to obtain a measure of the external angular rate. Each of the signals is multiplied by $\sin \left(\Delta \phi_{y z}\right), \sin \left(\Delta \phi_{x z}\right)$ and $\sin \left(\Delta \phi_{x y}\right)$, thus obtaining from equations (31):

$$
\begin{aligned}
& \frac{\partial \omega_{\Sigma}}{\partial \Omega_{x}}=\frac{\alpha_{y z}^{x}-\alpha_{z y}^{x}}{2}\left(1+\cos \left(2 \Delta \phi_{y z}\right)\right), \\
& \frac{\partial \omega_{\Sigma}}{\partial \Omega_{y}}=\frac{-\alpha_{z x}^{y}+\alpha_{x z}^{y}}{2}\left(1+\cos \left(2 \Delta \phi_{x z}\right)\right), \\
& \frac{\partial \omega_{\Sigma}}{\partial \Omega_{z}}=\frac{\alpha_{x y}^{z}-\alpha_{y x}^{z}}{2}\left(1+\cos \left(2 \Delta \phi_{x y}\right)\right) .
\end{aligned}
$$

From equations (32) it is evident that there is a high frequency component and a stationary component in the resultant signal, a low-pass filter is then applied to extract the signal, which is proportional to the angular rate through a certain sensitivity that reads:

$$
\begin{aligned}
& \frac{\partial \omega_{\Sigma}}{\partial \Omega_{x}}=\frac{\alpha_{y z}^{x}-\alpha_{z y}^{x}}{2}, \\
& \frac{\partial \omega_{\Sigma}}{\partial \Omega_{y}}=\frac{-\alpha_{z x}^{y}+\alpha_{x z}^{y}}{2}, \\
& \frac{\partial \omega_{\Sigma}}{\partial \Omega_{z}}=\frac{\alpha_{x y}^{z}-\alpha_{y x}^{z}}{2} .
\end{aligned}
$$

If the relation (5) is now substituted into equations (33), the unitary scale factors of an ideal gyroscope are obtained as expected:

$$
\begin{aligned}
& \frac{\partial \omega_{\Sigma}}{\partial \Omega_{x}}=-1, \\
& \frac{\partial \omega_{\Sigma}}{\partial \Omega_{y}}=1, \\
& \frac{\partial \omega_{\Sigma}}{\partial \Omega_{z}}=-1 .
\end{aligned}
$$


Equations (33) also give the scale factor for a distributed-mass gyroscope if the angular gains defined by equation (13) are used instead of those defined

modes. For this reason, the scale factors of a three-axial FM gyroscope are little dependent on over etch and other fabrication imperfections and are completely independent from environmental fluctuations such as temperature. This prop- 
erty represents the main advantage of the FM gyroscope with respect to the

\section{Mechanical Design}

The proof mass of a three-axial FM gyroscope must be able to simultaneously translate along the three orthogonal directions. Proper deformable portions and auxiliary structures must, then, be designed in order to guarantee the wanted motion of the gyroscope's proof mass and a good decoupling between the three modes of interest. In order to reject the effect of inertia forces possibly coming from an external acceleration and for a better functioning of the gyroscope as explained in Section 5.2, a fully differential two-masses structure is here proposed.

The introduction of a second mass actuated in an antiphase mode in the mechanical structure of the three-axial gyroscope allows the rejection of any unwanted common-mode contribution due e.g. to the external acceleration (see [19] for more details).

In Figure 3, a schematic and simplified view of the three modes of a FM three-axial gyroscope compatible with the surface micromachining fabrication process is shown. Note that surface micromachining processes do not allow the exploitation of fully three-dimensional mechanical designs: in-plane structures are in fact extruded in the $z$ - direction and consequently a uniform out-of-plane thickness, with a value fixed by the technological process, must be considered. The mechanical structure must then be properly designed to overcome such strict limitation of the fabrication process especially for the out-of-plane modes.

In this paper we show an innovative mechanical design that fullfils all the requirements of the FM working principle and respects the fabrication process constraints. In Figure 3, a schematic view of the proposed structure is shown. of freedom as whished, while the external frames have only one degree of freedom (indicated by the pink arrows in Figure 3) to allow for a good decoupling between 
the modes.
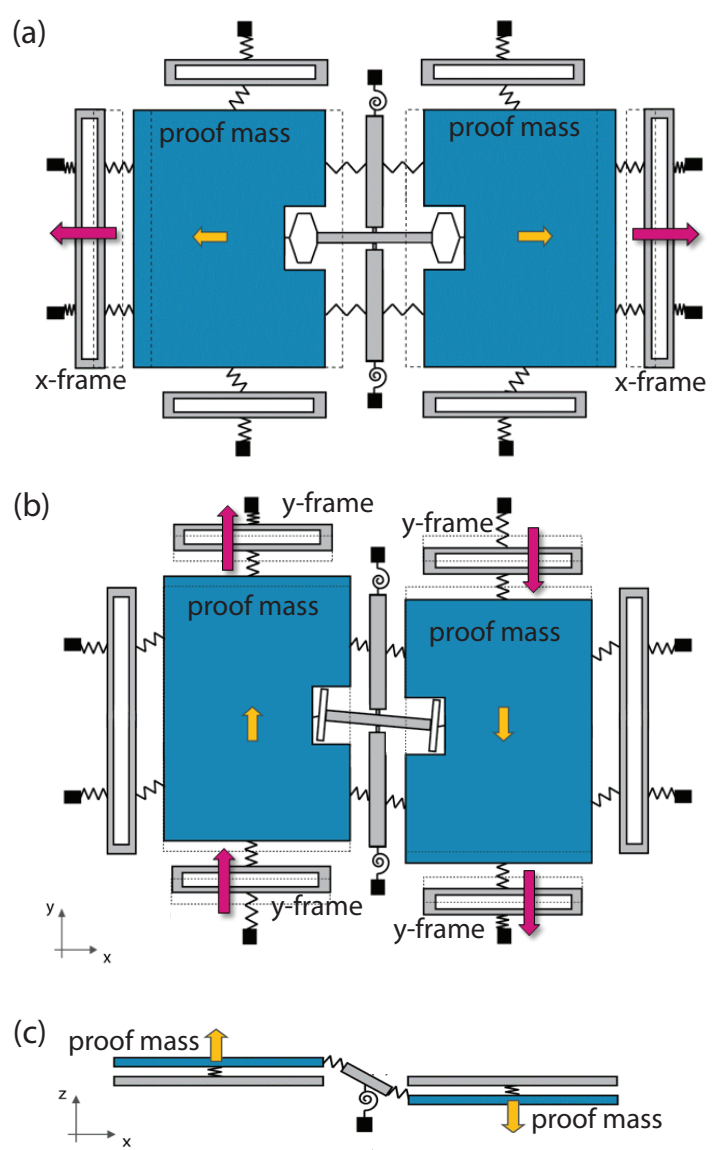

Figure 3: Schematic view of the three-axial FM gyroscope. Differential mode along the (a) $x$-axis, (b) $y$-axis and (c) $z$-axis.

A central cross-shaped auxiliary structure is added to allow the anti-phase motion of the two proof masses along the three orthogonal directions. The deformation of the springs connecting the central structure to the two proof masses allows the differential $x$-mode (see Figure $3 \mathrm{a}$ ) while the in-plane rotation of the horizontal central bridge (see Figure $3 \mathrm{~b}$ ) allows the differential $y$-mode. Finally, the out-of-plane torsional motion of the full cross-shaped auxiliary mass allows the differential $z$-mode (see Figure 3c). The black squares in Figure 3 represent the anchors to the substrate. In surface micromachining processes, in 
fact, the structures are suspended through springs and are at a fixed distance from the substrate. As it will be clearer later, this is another constraint that the MEMS designer must take into account during the design process.

\subsection{Implementation of the three-axial FM gyroscope}

Starting from the schematic view shown in Figure 3, a real design of a three-axial FM gyroscope compatible with the Thelma ${ }^{\circledR}$ surface micromachining process of STMicroelectronics (see [23] for more details) has been studied.

In Figure 4, an in-plane view of the mechanical design of the proposed threeaxial FM gyroscope is represented: it is possible to recognize the two proof masses, the external frames and the central cross-shaped auxiliary structure composed by two torsional masses and a central bridge. Figure 5 shows the shapes of the three anti-phase translational modes of the gyroscope structure.

The central bridge that can rotate around the out-of-plane $z$-axis with respect to the torsional masses thanks to the two hinge-like connections (see Figure 4 ), allows the differential motion of the proof masses of the gyroscope along the $y$-axis direction (see Figure 5a). Note that hinge-like connections must be obtained through short thin beams since it is not possible to obtain such kind of connections in standard MEMS fabrication processes.

The springs connecting the central bridge with the proof masses are mainly responsible of the anti-phase motion of the masses along the $x$-axis (see Figure $5 b)$.

The two auxiliary torsional masses are anchored to the substrate through torsional springs (two elongated beams located in the middle of the torsional masses and one folded spring sited on top (bottom) of the mass as shown in Figures 4e-f) that allow their rotation around the $y$-axis thus coupling, in a differential way, the two proof masses of the gyroscope during the out-of-plane mode (see Figure 5c). The out-of-plane translational motion of the proof masses is then achieved thus overcoming one of the major constraint of the surface micromachining process. Thanks to this shrewdness, in fact, we obtain an innovative mechanical structure able to translate in the $z$ - direction: on the contrary, 

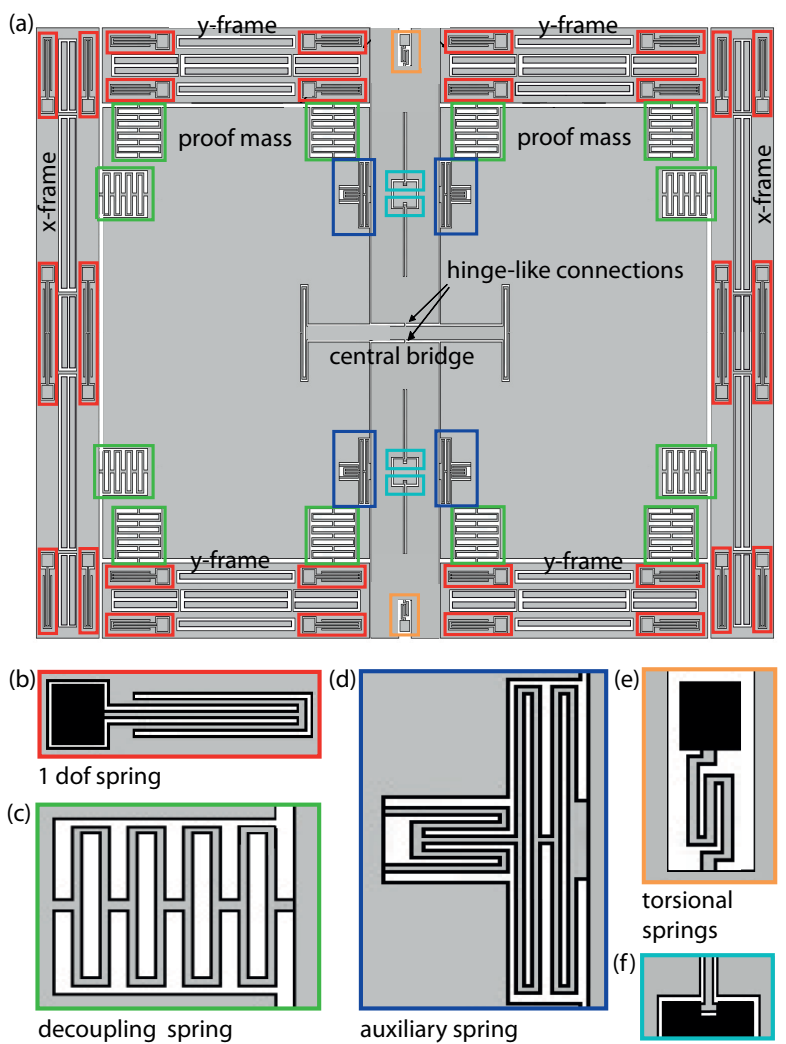

Figure 4: Schematic in-plane view of the three-axial FM gyroscope.

almost all the MEMS devices available so far exploit the torsional modes for the out-of-plane driving/sensing.

From Figure 5 one can observe that the proof masses of the three-axial FM gyroscope are well decoupled from the external frames containing the in-plane electrodes thanks to the ring-like springs shown in Figure 4c (see [19] for more details on the working principle of such spring). The displacement of the $x$-axis ( $y$-axis) external frames during the $y$-mode ( $x$-mode) is less than $1 / 10(1 / 19)$ of the motion of the proof masses while the displacement of the external frames during the $z$-mode of the device is less than $1 / 30$ of the out-of-plane motion of the two proof masses. The suspending springs connecting the external frames to the substrate (see Figure $4 \mathrm{~b}$ ) allow only one degree of freedom and consequently 
(a)

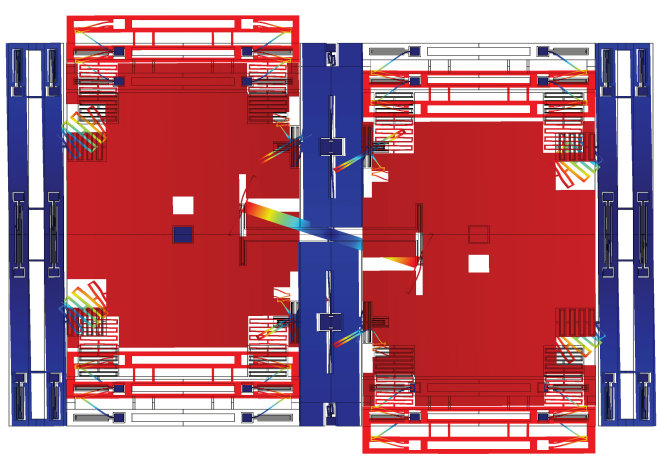

(b)

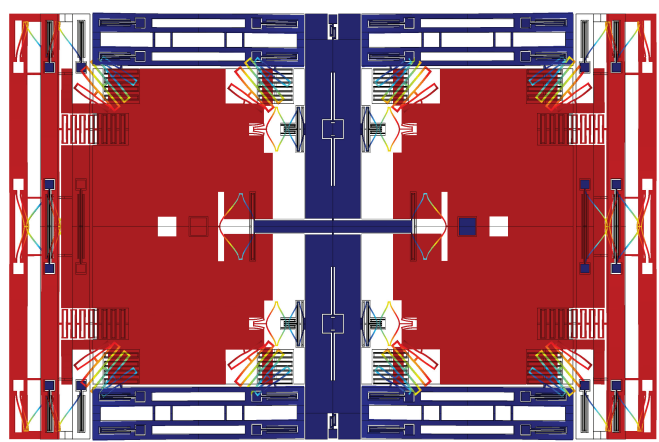

(c)

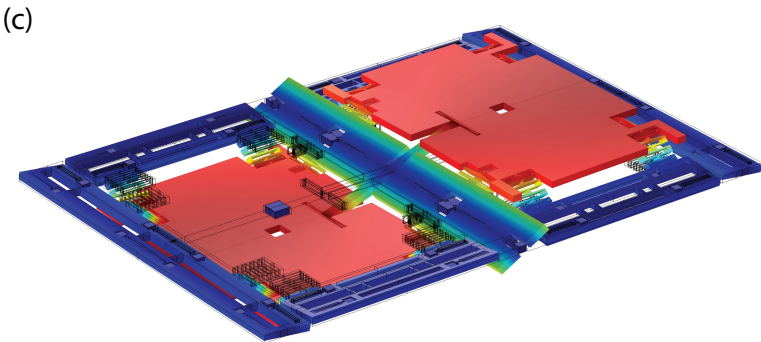

Figure 5: Modal analysis of the three-axial FM gyroscope performed in COMSOLMultiphysics $^{\circledR}$. The contour of the magnitude of the displacement field is shown in color. Only the three differential translational modes of interest are shown: a) $y$-axis mode $(f=44087 \mathrm{~Hz}), \mathrm{b}) x$-axis mode $(f=44150 \mathrm{~Hz}), \mathrm{c}) z$-axis mode $(f=45712 \mathrm{~Hz})$. 


\begin{tabular}{|c|c|c|c|}
\hline & IPx mode & IPy mode & OP mode \\
\hline Frequency & $44150 \mathrm{~Hz}$ & $44087 \mathrm{~Hz}$ & $45712 \mathrm{~Hz}$ \\
Stiffness & $3295 \mathrm{~N} / \mathrm{m}$ & $3127 \mathrm{~N} / \mathrm{m}$ & $2921 \mathrm{~N} / \mathrm{m}$ \\
Modal Mass & $39 \mathrm{nKg}$ & $38 \mathrm{nKg}$ & $33 \mathrm{nKg}$ \\
Quality factor & 7000 & 7500 & 950 \\
\hline
\end{tabular}

Table 1: Main mechanical properties of both the in-plane (IPx and IPy) and out-of-plane (OP) modes of the three-axial FM gyroscope shown in Figure 4.

improve the decoupling between the modes.

In Table 1 the main mechanical properties computed though Finite Element 370 (FE) analyses for a standard over etch, are summarized. The quality factor estimation is carried out through the numerical code based on the Boundary Integral Equation approach presented in [24] and [25].

The modes have been designed such as to guarantee comparable stiffness and modal mass for the three axes. Despite the fabrication process forces a completely different geometry in-plane and out-of-plane, a proper design of the springs and the masses allows to obtain similar mechanical properties for the three modes of interest. This is fundamental in such devices where the scale factor only depends on the modal masses involved in the modes. The expected sensitivity can be calculated from equations (33) and (13) and is equal to 0.92 , 0.91 and 0.84 for $x, y$ and $z$ respectively.

The difference in terms of quality factor is actually very difficult to reduce through the mechanical design, due to fabrication process constraints: a proper sizing of the driving electrodes is required to obtain similar displacement amplitudes.

\subsection{Electrostatic actuation/detection schemes}

In MEMS gyroscopes the modes of interest are usually actuated (driven in MEMS language) through electrostatic forces thanks to parallel plate or comb fingers schemes. Moreover, a differential readout and a push-pull actuation 
scheme are usually desirable in all the three orthogonal directions with the purpose to improve the performance of the device: these schemes mainly consist on placing electrodes on the two sides of the movable mass thus doubling the driving signal and rejecting common mode unwanted sensed signals.

Differently from what happens for common three-axial AM gyroscopes where two driven axes are required, in FM gyroscopes three translational modes must be actuated through electrostatic forces: proper electrodes must be then designed to allow the motion of the proof mass along the three orthogonal directions.

In the out-of-plane direction, there are several limitations due to the fabrication process such as the above mentioned fixed thickness of the structure and the possibility to have only bottom electrodes at a fixed distance (e.g. $1.8 \mu \mathrm{m})$ from the proof mass. Due to these constraints, driving motion along the out-of-plane axis must be exclusively provided by a parallel plate scheme. For the sake of similarity among all the axes, a parallel plate actuation/detection scheme is also used for the other two driving axes of the three-axial FM gyroscope. A target displacement of $170 \mathrm{~nm}$ is chosen for all the axes of the proposed design as a compromise between the linear electrostatic behavior and the noise performance (see [21]) of the device.

In Figure 6 the electrodes employed for both driving and sensing along the three directions are shown. The electrodes for the driving and sensing of the motion along the $x(y)$-axis are sited inside the two properly decoupled frames located on the left and right (top and bottom) sides of the proof masses. The electrodes located on the substrate are shown in light blue over the proof masses: they are responsible of the driving and sensing of the out-of-plane motion. The symmetry of these electrodes has the purpose to facilitate the out-of-plane translation of the gyroscope's proof masses avoiding any torsional movements.

Moreover, additional tuning electrodes are added in the $z$-direction in order to compensate the mismatch between the in-plane and the out-of-plane modes that can arise after the fabrication process. The unknown over etch of the process in fact usually causes the deviation of the mismatch between the different 


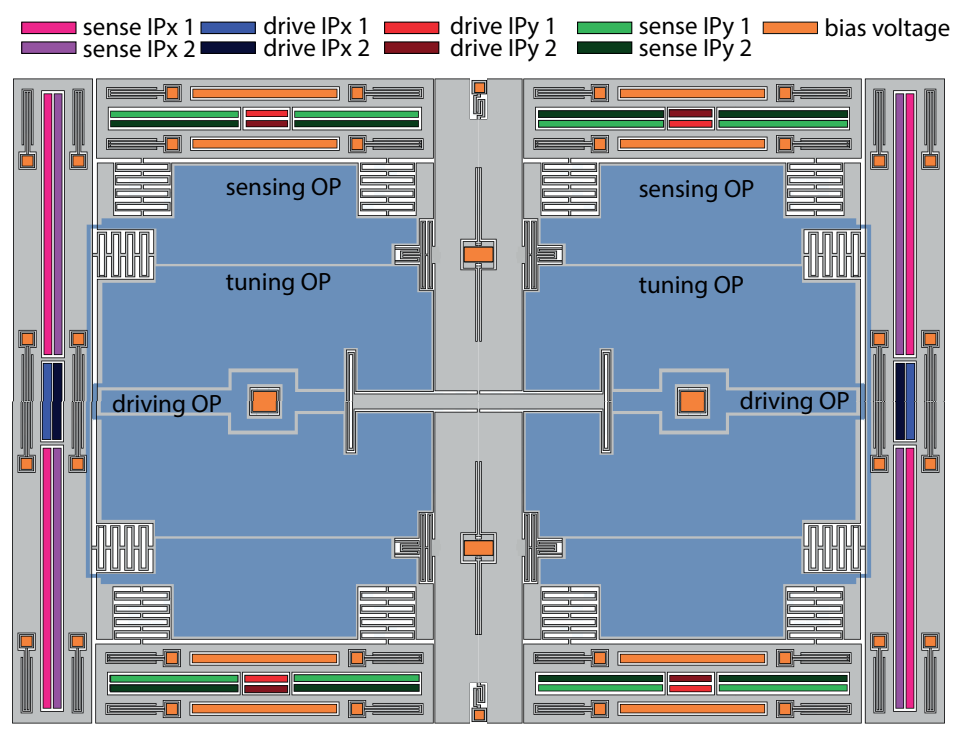

Figure 6: Schematic in-plane view of both the in-plane (IPx and IPy) and out-of-plane (OP) electrodes for the three-axial FM gyroscope shown in Figure 4.

modes from the nominal value computed through simulation with a standard over etch of $0.35 \mu \mathrm{m}$ : the electrostatic softening induced by the presence of tuning electrodes kept at a fixed voltage is exploited to compensate such variation. In the proposed realization, tuning electrodes are sized such as to reach a tuning up to $2000 \mathrm{~Hz}$ of the out-of-plane mode for a maximum voltage of $23 \mathrm{~V}$. Note also that the out-of-plane mode is intentionally designed at higher frequency with respect to the two in-plane ones for this reason (see Figure 5).

Finally, thanks to the two-masses structure, the differential readout and the push-pull actuation schemes are achieved also in the out-of-plane direction where the electrodes can be sited only on the substrate and not on both the cap and the substrate. An important fabrication constraint is then overcame through the proposed innovative mechanical design.

\section{Experimental characterization}

A prototype of the FM three-axial gyroscope shown in Figure 4 has been fabricated through the ThELMA ${ }^{\circledR}$ surface micromachining process developed 

image of the fabricated device is shown. The overall footprint $(1089 \mu \mathrm{m} \times 1595$ $\mu \mathrm{m})$ is comparable with the one of the AM three-axial gyroscopes actually on the market (see e.g. [3]).

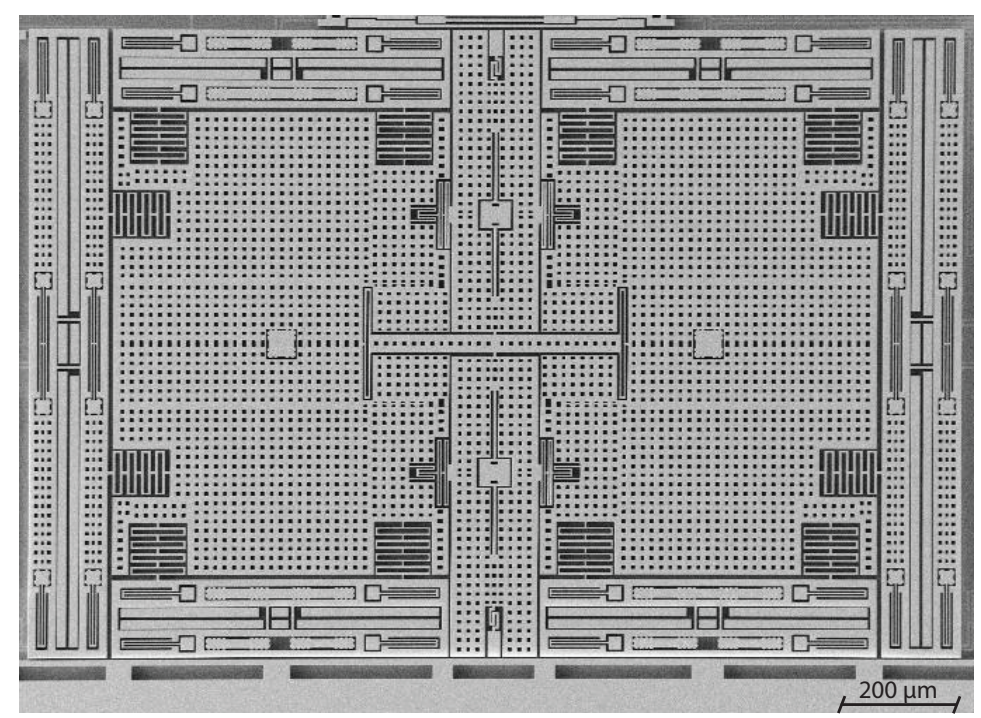

Figure 7: SEM image of the fabricated three-axial FM gyroscope.

A first preliminary mechanical characterization of the fabricated device has been carried out and in Figure 8 the frequency responses of the three modes of the FM gyroscope are reported. In Table 2, the experimental values of the main mechanical properties of the structure are reported. A good agreement with the design values of Table 1 is found in terms of both natural frequencies and quality factor, thus proving the validity of the simulation tools adopted. The slight difference in terms of natural frequency is due to the different over etch obtained after the fabrication process while the difference in terms of quality factor can be attributed to both the unknown process parameters: the over etch and the pressure inside the package. 


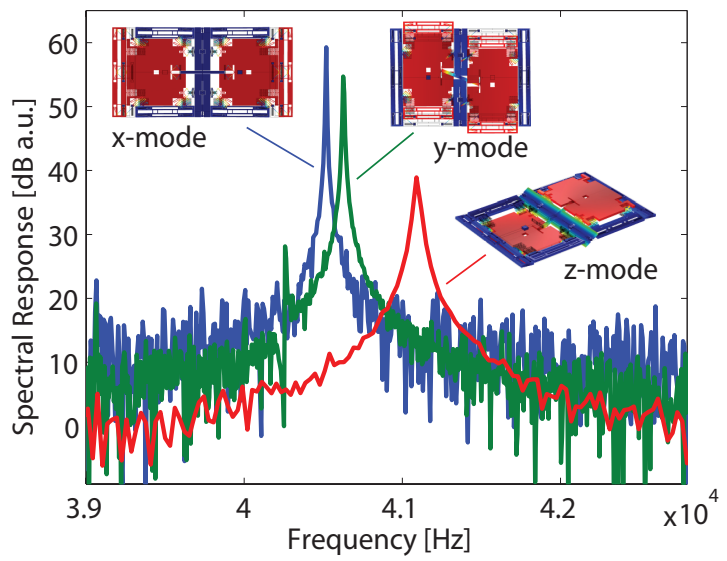

Figure 8: Experimental frequency response of the fabricated three-axial FM gyroscope shown in Figure 7.

\begin{tabular}{|c|c|c|c|}
\hline & IPx mode & IPy mode & OP mode \\
\hline Frequency & $40528 \mathrm{~Hz}$ & $40627 \mathrm{~Hz}$ & $41150 \mathrm{~Hz}$ \\
Quality factor & 6598 & 5117 & 1133 \\
\hline
\end{tabular}

Table 2: Experimental measurements of the main mechanical properties of both the in-plane (IPx and IPy) and out-of-plane (OP) modes of the three-axial FM gyroscope shown in Figure 4.

\section{Conclusions}

The working principle of a three-axial FM MEMS gyroscope is described in this paper through a theoretical model that takes into account also non-ideal terms coming from fabrication imperfections.

The proposed design (see Figure 4) represents the first three-axial gyroscope designed to work in FM operating mode (see [18]). It is a simple structure that, with only two masses, guarantees the differential actuation and readout of three axes. With a relative small footprint $(1090 \mu \mathrm{m} \times 1595 \mu \mathrm{m})$ it can, in fact, measure three different angular velocities acting along the three orthogonal directions $(x-, y$ - and $z$-). 
A good decoupling between the modes is, moreover, provided by the presence

460 is expected being the modal masses involved in the three modes very close to each others.

Preliminary experimental characterizations proves the validity of the mechanical design and of the simulation tools employed.

465 design with two masses able to translate along the three orthogonal directions (also along the $z$-direction) thanks to a push-pull actuation scheme and with a differential readout (also along the $z$-direction). The present design, therefore, overcame the limitations of the fabrication process and presents an out-of-plane

[1] A. Corigliano, R. Ardito, C. Comi, A. Frangi, A. Ghisi, S. Mariani, Mechanics of Microsystems, 2018, Wiley, ISBN 978-1-119-05383-5.

[2] C. Acar, A. Shkel, MEMS vibratory gyroscopes. Structural approaches to improve robustness, 2009, Springer.

480

mode similar, in properties, to the other in-plane modes (i.e. push-pull, differential reading, translation).

The authors are currently working on the coupling of the fabricated device with the Application Specific Integrated Circuit (ASIC) presented in [16] in order to perform sensitivity measurements.

\section{References}

3] B. Vigna, Tri-axial mems gyroscopes and six degree-of-freedom motion sensors, in: IEEE International Electron Devices Meeting, Washington DC, USA, 2011, pp. 29.1.1-3.

[4] A. Esmaeili, M. A. Kupaei, H. Faghihian, H. R. Mirdamadi, An adaptable broadband mems vibratory gyroscope by simultaneous optimization of robustness and sensitivity parameters, Sensors 
and Actuators A: Physical 206 (Supplement C) (2014) 132 - 137. doi:doi.org/10.1016/j.sna.2013.12.014.

[5] G. Wu, G. Chua, Y. Gu, A dual-mass fully decoupled mems gyroscope with wide bandwidth and high linearity, Sensors and Actuators A: Physical 259 (2017) 50 - 56. doi:https://doi.org/10.1016/j.sna.2017.03.027.

[6] P. Taheri-Tehrani, M. Kline, I. Izyumin, B. Eminoglu, Y. C. Yeh, Y. Yang, Y. Chen, I. Flader, E. J. Ng, T. W. Kenny, B. E. Boser, D. A. Horsley, Epitaxially-encapsulated quad mass gyroscope with nonlinearity compensation, in: IEEE 29th International Conference on Micro Electro Mechanical Systems (MEMS), 2016, pp. 966-969. doi:10.1109/MEMSYS. 2016.7421793.

[7] S. Sonmezoglu, S. E. Alper, T. Akin, An automatically modematched mems gyroscope with wide and tunable bandwidth, Journal of Microelectromechanical Systems $23 \quad$ (2) (2014) 284-297. doi : 10.1109/JMEMS . 2014.2299234.

[8] E. Tatar, S. E. Alper, T. Akin, Quadrature-error compensation and corresponding effects on the performance of fully decoupled mems gyroscopes, Journal of Microelectromechanical Systems 21 (3) (2012) 656-667. doi : 10.1109/JMEMS . 2012. 2189356.

[9] S. Sonmezoglu, P. Taheri-Tehrani, C. Valzasina, L. G. Falorni, S. Zerbini, S. Nitzan, D. A. Horsley, Single-structure micromachined three-axis gyroscope with reduced drive-force coupling, IEEE Electron Device Letters 36 (9) (2015) 953-956. doi:10.1109/LED.2015.2454511.

[10] E. Tatar, T. Mukherjee, G. K. Fedder, Stress effects and compensation of bias drift in a mems vibratory-rate gyroscope, Journal of Microelectromechanical Systems $26 \quad(3) \quad(2017) \quad 569-579$. doi:10.1109/JMEMS . 2017.2675452. 
[11] M. H. Kline, Y.-C. Yeh, B. Eminoglu, I. Izyumin, M. Daneman, D. A. Horsley, B. E. Boser, Mems gyroscope bias drift cancellation using continuoustime mode reversal, in: The 17th International Conference on Transducers \& Eurosensors XXVII, Barcelona, Spain, 2013, pp. 1855-1858. doi:http://dx.doi.org/10.1109/Transducers.2013.6627152.

[12] B. Eminoglu, Y.-C. Yeh, I. Izyumin, I. Nacita, M. Wireman, A. Reinelt, B. E. Boser, Comparison of long-term stability of am versus fm gyroscopes, in: IEEE 29th International Conference on Micro Electro Mechanical Systems (MEMS), Shanghai, China, 2016, pp. 954-957.

[13] M. H. Kline, Y.-C. Yeh, B. Eminoglu, H. Najar, M. Daneman, D. A. Horsley, B. E. Boser, Quadrature fm gyroscope, in: IEEE 26th International Conference on Micro Electro Mechanical Systems (MEMS), Taipei, Taiwan, 2013, pp. 604-608. doi:http://dx.doi.org/10.1109/MEMSYS2013.6474314.

[14] I. Izyumin, M. H. Kline, Y.-C. Yeh, B. Eminoglu, C. H. Ahn, V. A. Hong, Y. Yang, E. J. Ng, T. W. Kenny, B. E. Boser, A 7ppm, 6 hr frequencyoutput mems gyroscope, in: IEEE 28th International Conference on Micro Electro Mechanical Systems (MEMS), Estoril, Portugal, 2015, pp. 33-36. doi:http://dx.doi.org/10.1109/MEMSYS.2015.7050879.

[15] V. Zega, P. Minotti, G. Mussi, A. Tocchio, L. Falorni, S. Facchinetti, A. Bonfanti, A. L. Lacaita, C. Comi, G. Langfelder, A. Corigliano, The first frequency modulated (fm) pitch gyroscope, in: Proceedings 2017, Vol. 1, 2017, p. 393.

[16] P. Minotti, S. Dellea, G. Mussi, A. Bonfanti, S. Facchinetti, A. Tocchio, V. Zega, C. Comi, A. L. Lacaita, G. Langfelder, High scale-factor stability frequency-modulated mems gyroscope: 3-axis sensor and integrated electronics design, IEEE Transactions on Industrial Electronics (2017) in pressdoi:10.1109/TIE. 2017.2772212. 
[17] I. Izyumin, B. E. Boser, M. H. Kline, Frequency readout gyroscope, Patent wo/2014/093727 (2014).

[18] A. Tocchio, L. Falorni, C. Comi, V. Zega, Giroscopio triassiale mems a modulazione di frequenza, deposited patent n.: 102016000106928 (2016).

[19] V. Zega, C. Comi, P. Minotti, L. Falorni, A. Tocchio, G. Langfelder, A. Corigliano, Mechanical design of a fully-differential triaxial frequency modulated mems gyroscope, in: AIMETA 2017 - Proceedings of the XXIII Conference of the Italian Association of Theoretical and Applied Mechanics, Vol. 1, 2017, pp. 420-427.

[20] Geogebra, https://www.geogebra.org, accessed: 2017-10-25.

[21] P. Minotti, G. Mussi, S. Dellea, C. Comi, V. Zega, S. Facchinetti, A. Tocchio, A. Bonfanti, G. Langfelder, A $160 \mu \mathrm{a}, 8 \mathrm{mdps} / \mathrm{hz}^{1 / 2}$ frequencymodulated mems gyroscope, in: Inertial 2017, Kauai, Hawaii, 2017.

[22] R. Mirzazadeh, S. E. Azam, E. Jansen, S. Mariani, Uncertainty quantification in polysilicon mems through on-chip testing and reduced-order modelling, in: 2017 18th International Conference on Thermal, Mechanical and Multi-Physics Simulation and Experiments in Microelectronics and Microsystems (EuroSimE), 2017, pp. 1-8.

[23] A. Corigliano, B. De Masi, A. Frangi, C. Comi, A. Villa, M. Marchi, Mechanical characterization of polysilicon through onchip tensile tests, J. Microelectromech. Syst. 13 (2) (2004) 200-219. doi:10.1109/JMEMS . 2003.823221.

[24] A. Frangi, P. Fedeli, G. Laghi, G. Langfelder, G. Gattere, Near vacuum gas damping in mems: Numerical modeling and experimental validation, J. Microelectromech. Syst. 25 (5) (2016) 890-899. doi:10.1109/JMEMS . 2016. 2584699. 
[25] P. Fedeli, A. Frangi, G. Laghi, G. Langfelder, G. Gattere, Near vacuum gas damping in mems: Simplified modeling, Journal of Microelectromechanical Systems 26 (3) (2017) 632-642. doi:10.1109/JMEMS . 2017. 2686650.

\section{Appendix A.}

The expressions for the variation of the amplitudes of motion for an ideal gyroscope read:

$$
\begin{aligned}
\dot{A}_{x}= & -\frac{b_{x}}{2 m} A_{x}+\beta_{x y}^{x y} \Omega_{x} \Omega_{y} \frac{A_{y}}{2 \omega_{x}} \sin \left(\Delta \phi_{x y}\right)+ \\
& +\beta_{x z}^{x z} \Omega_{x} \Omega_{z} \frac{A_{z}}{2 \omega_{x}} \sin \left(\Delta \phi_{x z}\right)-\alpha_{x y}^{z} \Omega_{z} \frac{\omega_{y}}{\omega_{x}} A_{y} \cos \left(\Delta \phi_{x y}\right)+ \\
& -\alpha_{x z}^{y} \Omega_{y} \frac{\omega_{z}}{\omega_{x}} A_{z} \cos \left(\Delta \phi_{x z}\right)+\frac{\bar{F}_{x}}{2 m \omega_{x}}, \\
\dot{A}_{y}= & -\frac{b_{y}}{2 m} A_{y}-\beta_{y x}^{y x} \Omega_{x} \Omega_{y} \frac{A_{x}}{2 \omega_{y}} \sin \left(\Delta \phi_{x y}\right)+ \\
& +\beta_{y z}^{y z} \Omega_{y} \Omega_{z} \frac{A_{z}}{2 \omega_{y}} \sin \left(\Delta \phi_{y z}\right)-\alpha_{y x}^{z} \Omega_{z} \frac{\omega_{x}}{\omega_{y}} A_{x} \cos \left(\Delta \phi_{x y}\right)+ \\
& -\alpha_{y z}^{x} \Omega_{x} \frac{\omega_{z}}{\omega_{y}} A_{z} \cos \left(\Delta \phi_{y z}\right)+\frac{\bar{F}_{y}}{2 m \omega_{y}}, \\
\dot{A}_{z}= & -\frac{b_{z}}{2 m} A_{z}-\beta_{z x}^{z x} \Omega_{x} \Omega_{z} \frac{A_{x}}{2 \omega_{z}} \sin \left(\Delta \phi_{x z}\right)+ \\
& -\beta_{z y}^{z y} \Omega_{y} \Omega_{z} \frac{A_{y}}{2 \omega_{z}} \sin \left(\Delta \phi_{y z}\right)-\alpha_{z x}^{y} \Omega_{y} \frac{\omega_{x}}{\omega_{z}} A_{x} \cos \left(\Delta \phi_{x z}\right)+ \\
& -\alpha_{z y}^{x} \Omega_{x} \frac{\omega_{y}}{\omega_{z}} A_{y} \cos \left(\Delta \phi_{y z}\right)+\frac{\bar{F}_{z}}{2 m \omega_{z}},
\end{aligned}
$$

575 while for a real gyroscope, they read:

$$
\begin{aligned}
\dot{A}_{x}= & \left(\dot{A}_{x}\right)_{\text {ideal }}+\frac{k_{x y}}{2 \omega_{x} m_{x}} A_{y} \sin \left(\Delta \phi_{x y}\right)+ \\
& +\frac{k_{x z}}{2 \omega_{x} m_{x}} A_{z} \sin \left(\Delta \phi_{x z}\right)-\frac{b_{x y}}{2 m_{x}} \frac{\omega_{y}}{\omega_{x}} A_{y} \cos \left(\Delta \phi_{x y}\right)+ \\
& -\frac{b_{x z}}{2 m_{x}} \frac{\omega_{z}}{\omega_{x}} A_{z} \cos \left(\Delta \phi_{x z}\right)
\end{aligned}
$$




$$
\begin{aligned}
\dot{A}_{y}= & \left(\dot{A}_{y}\right)_{\text {ideal }}-\frac{k_{y x}}{2 \omega_{y} m_{y}} A_{x} \sin \left(\Delta \phi_{x y}\right)+ \\
& +\frac{k_{y z}}{2 \omega_{y} m_{y}} A_{z} \sin \left(\Delta \phi_{y z}\right)-\frac{b_{y x}}{2 m_{y}} \frac{\omega_{x}}{\omega_{y}} A_{x} \cos \left(\Delta \phi_{x y}\right)+ \\
& -\frac{b_{y z}}{2 m_{y}} \frac{\omega_{z}}{\omega_{y}} A_{z} \cos \left(\Delta \phi_{y z}\right), \\
\dot{A}_{z}= & \left(\dot{A}_{z}\right)_{i d e a l}-\frac{k_{z y}}{2 \omega_{z} m_{z}} A_{y} \sin \left(\Delta \phi_{y z}\right)+ \\
& -\frac{k_{z x}}{2 \omega_{z} m_{z}} A_{x} \sin \left(\Delta \phi_{x z}\right)-\frac{b_{z x}}{2 m_{z}} \frac{\omega_{x}}{\omega_{z}} A_{x} \cos \left(\Delta \phi_{x z}\right)+ \\
& -\frac{b_{z y}}{2 m_{z}} \frac{\omega_{y}}{\omega_{z}} A_{y} \cos \left(\Delta \phi_{y z}\right),
\end{aligned}
$$

where $\left(\dot{A}_{i}\right)_{\text {ideal }}$ are given by equations (A.1)-(A.3) with $m_{i}$ instead of $m$. Note that the forcing terms $\bar{F}_{i}$ are fundamental to guarantee the control on the velocities amplitudes (28) required by the FM working principle. 